\title{
Correlation of 1.5 Tesla Magnetic Resonance Imaging with Clinical and Intraoperative Findings for Lumbar Disc Herniation
}

\author{
Shumayou Dutta, Arvind Bhave, Sanjay Patil \\ Department of Orthopaedics, Bharati Vidyapeeth Deemed Hospital and Medical College, Pune, India
}

\begin{abstract}
Study Design: A single-center prospective study.
Purpose: A magnetic resonance imaging (MRI) scan is undeniably the gold standard for the diagnosis of a lumbar disc prolapse. Unfortunately it shares a strong association with incidental findings. In this study, we aimed to determine the extent to which a 1.5 Tesla MRI correlates with the clinical features and intraoperative findings in cases of lumbar disc prolapse.

Overview of Literature: Few studies have correlated MRI with clinical findings, and none have extended this correlation to intraoperative findings.

Methods: Over a 2-year period, 50 consecutive patients with lumbar disc herniation requiring discectomy were studied. The MRI findings we observed consisted of the prolapse level, type, position, migration, high-intensity zones (HIZ), lateral recess, and foraminal stenosis. A logistic regression analysis was performed to determine the significance for the various MRI findings. Finally, the MRI observations were confirmed with intraoperative findings and inferences were drawn.

Results: MRI scan sensitivity and specificity for determining surgically significant levels was $100 \%$ and $94.94 \%$, respectively. Straight leg raising test was positive in $74 \%$ of patients, with $85 \%, 43 \%$, and $75 \%$ for paracentral, central, and foraminal levels, respectively. A foraminal compromise was the only MRI parameter to share a significant association with neurological deficits. Patients with a HIZ on the MRI had a significant increase in back pain and 63\% exhibited identifiable annular tears intraoperatively. The intraoperative anatomical findings correlated extensively with the MRI findings.

Conclusions: MRI findings strongly correlate with intraoperative features and can serve as a useful tool when planning surgery due to the accurate depiction of the morphometric features. However, the decision for surgery should be made only when detailed clinical findings in conjunction with MRI findings allow for an accurate identification of the culprit fragment and pain generators.
\end{abstract}

Keywords: Lumbar; Prolapsed disc; Slipped disc; Sciatica; Magnetic resonance imaging; Correlation study; Intraoperative

\section{Introduction}

Lumbar disc prolapse is a common cause of back and leg pain affecting today's working population and is a major cause of sickness absenteeism, creating a substantial socio-economic burden [1,2]. The diagnostic and surgical management of lumbar disc prolapse have evolved over time, ranging from open surgery to minimal access surgeries, such as tubular-assisted discectomy and a percutaneous endoscopic lumbar discectomy. Progress in

Received Dec 30, 2015; Revised May 1, 2016; Accepted May 6, 2016

Corresponding author: Shumayou Dutta

Bharati Vidyapeeth Deemed Hospital and Medical College, Pune Satara Road, Pune, Maharashtra 411043, India

Tel: 91-020-24373226, Fax: +91-020-24373222, E-mail: shumayou@hotmail.com 
surgical techniques was aided by the advances made in imaging techniques which provide an accurate depiction of the local morphometry in each case. The computer tomography scan provided the first view of the spine but has since been replaced by magnetic resonance imaging (MRI), which is the gold standard for the diagnosis of lumbar disc prolapse owing to its superior ability to discriminate between disc tissue and the spinal cord, as well as reveal key information regarding other morphometric issues [3]. MRI scans are available in various magnetic field strengths, with 1.5 Tesla being the most commonly used in India. Several studies have reported on the high sensitivity of this imaging modality [4-8]. However, MRI studies in selected volunteers have also shown up to a $36 \%$ incidence of disc protrusion and extrusion in patients without symptoms, thus highlighting their low predictive value for the development of back and leg pain $[9,10]$. Therefore, it is important to define the correlation between MRI findings and clinical features, as well as to define their degree of correlation with intraoperative findings. Although some studies have correlated MRI scans with clinical findings $[6,11,12]$, to our knowledge there are none that have also included intraoperative findings. The aim of the present study was to determine the correlation of a 1.5 Tesla MRI scan with clinical and intraoperative findings in patients with lumbar disc herniation.

\section{Materials and Methods}

A prospective cohort study was conducted at a tertiarycare institution between July 2011 and June 2013. Over a two-year period, 50 consecutive patients with lumbar disc herniation requiring discectomy, were included in this study. Patients were considered surgical candidates only when a conservative trial of at least eight weeks had failed. This trial included a structured program of rest with analgesics followed by physiotherapy. The indications for surgery were (1) persistent pain unrelieved by conservative treatment (at least eight weeks), (2) deteriorating neurology, and (3) Cauda equina syndrome. Patients who had undergone a same/adjacent level surgery before and those with any other associated pathology, were excluded from the study.

All the patients were subjected to a 1.5 Tesla MRI scan. A standard protocol was followed for every case, in which the scan was conducted with a circularly polarized spine array coil placed under the lumbar spine. The scans were obtained for the axial and sagittal planes; axial sections were taken parallel across each of the lumbar discs and the superior and inferior endplates of the lumbar vertebrae with a slice thickness of $3 \mathrm{~mm}$. Disc prolapse on the MRI was designated as significant when compression of the thecal sac and adjoining nerve root was present and accompanied by associated clinical symptoms. The recorded MRI findings consisted of the prolapse level, position (central, paracentral, foraminal, and extraforaminal), type (bulge, protrusion, extrusion, and sequestration), migration (superior and inferior), high-intensity zones, lateral recess, and foraminal stenosis (thecal sac compression, nerve root contact, and nerve root compression [13]) These findings were reported by a senior radiologist. The clinical criteria used to evaluate these patients were (1) a low backache with radiation to the lower limb, (2) radicular pain along a specific dermatome, (3) straight leg raising test (SLRT) for nerve root tension, (4) the presence of a neurological deficit, and (5) gait.

Patients who fulfilled the inclusion criteria received an open discectomy. Intraoperative findings were noted regarding the type, position of the fragment, migration, annular tear, lateral recess, and foraminal stenosis. These observations were then compared to the MRI findings.

All statistical analyses were performed using SPSS ver. 22.0 (IBM Corp., Armonk, NY, USA). $p<0.05$ was set as significant. A logistic regression analysis was performed to study the significance of various MRI factors that corresponded with a neurologic deficit to determine the odd's ratio and clinical significance for each comparison.

\section{Results}

In this study, the mean patient age was 49 years (range, $22-69$ years), of which $58 \%(n=29)$ were men and $42 \%$ $(n=21)$ were women. A total of 50 patients were found to have 56 levels of disc herniation; 44 patients had a singlelevel lumbar disc prolapse and six had two-level prolapses. Distribution of the single-level lumbar disc herniation was as follows: 23 at L45, 18 at L5S1, and three at L34. In the two-level herniation group there were two patients with a L45 and L5S1 disc prolapse, two patients with a L34 and L45 disc prolapse, and two patients with a L34 and L5S1 disc prolapse. A disc prolapse was considered to be "clinically significant" when the clinical findings (e.g., left or right leg pain and dermatomal/myotome distribution of pain/weakness) positively correlated with the MRI 
findings. A total of 51 levels was deemed to be clinically significant after a thorough examination of the patient, and the patients underwent an open discectomy. With respect to determining clinically significant levels, this study revealed that out of the 56 levels of disc lesions, 51 were symptomatic, and five were asymptomatic (Figs. 1, 2). An MRI is a very sensitive test for identifying disc lesions but is not highly specific. All levels were correctly identified, and no incorrect level of exposure was performed.

For the MRI scans, the location of the 51 operated disc prolapse levels was studied. A total of 33 were found to be paracentral, 14 central, and four foraminal. Of these, $28(85 \%)$, six (43\%), and three (75\%), respectively, were associated with a positive SLRT. So, overall, the SLRT was positive (between $15^{\circ}$ to $75^{\circ}$ ) in 37 patients $(74 \%)$. The logistic regression analysis revealed no significant association between the grade of disc prolapse, migration and SLRT.

A neurological deficit was observed in 24 patients. Of these, 13 exhibited grade 4 power, eight had grade 3 , and three had grade 1 power. All myotomes were highly
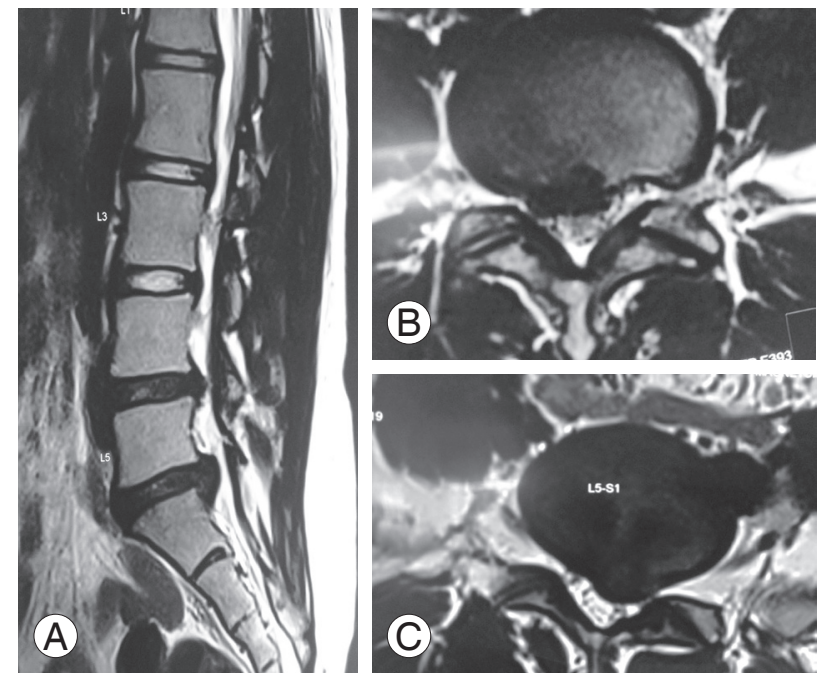

Fig. 1. (A) A T2-weighted sagittal magnetic resonance imaging of a 22-year-old girl complaining of left S1 radiculopathy exhibiting twolevel degeneration and herniation. (B) A T2-weighted axial scan revealing a $L 45$ right paracentral herniation. (C) T2-weighted axial scan at the L5S1 level of the same patient showing a left paracentral herniation compressing the S1 root. This was significant as it correlated with the clinical findings and a discectomy was performed at this level. The patient experienced a complete relief of her symptoms.

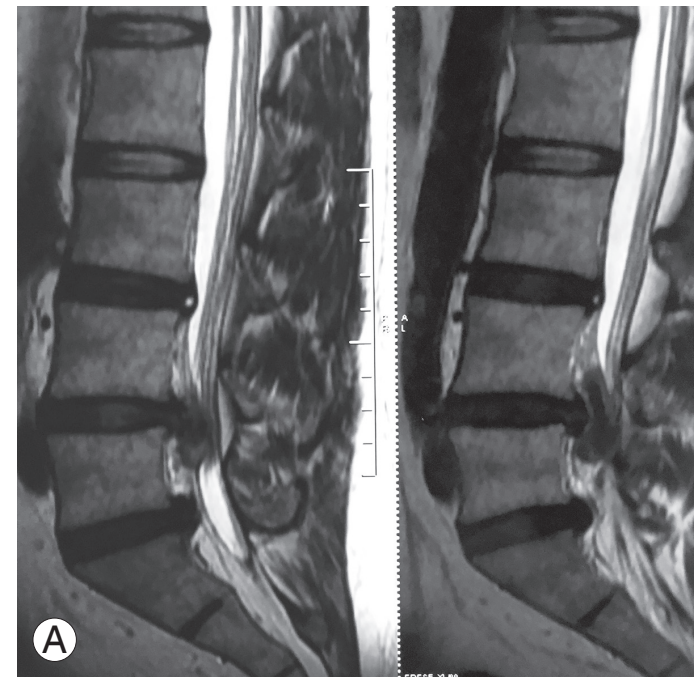

Fig. 2. (A) A 35-year-old male patient, symptomatic for left $\mathrm{L} 5$ radiculopathy. $\mathrm{A} T 2$-weighted sagittal magnetic resonance imaging showing a herniation at the L45 and L5S1 level. (B) A T2-weighted axial scan of the L45 and L5S1 levels. A detailed clinical examination revealed the $L 45$ to be clinically significant and L5S1 to be incidental. The patient was operated on for a $L 45$ disc prolapse. Postsurgery he experienced complete relief from his symptoms.

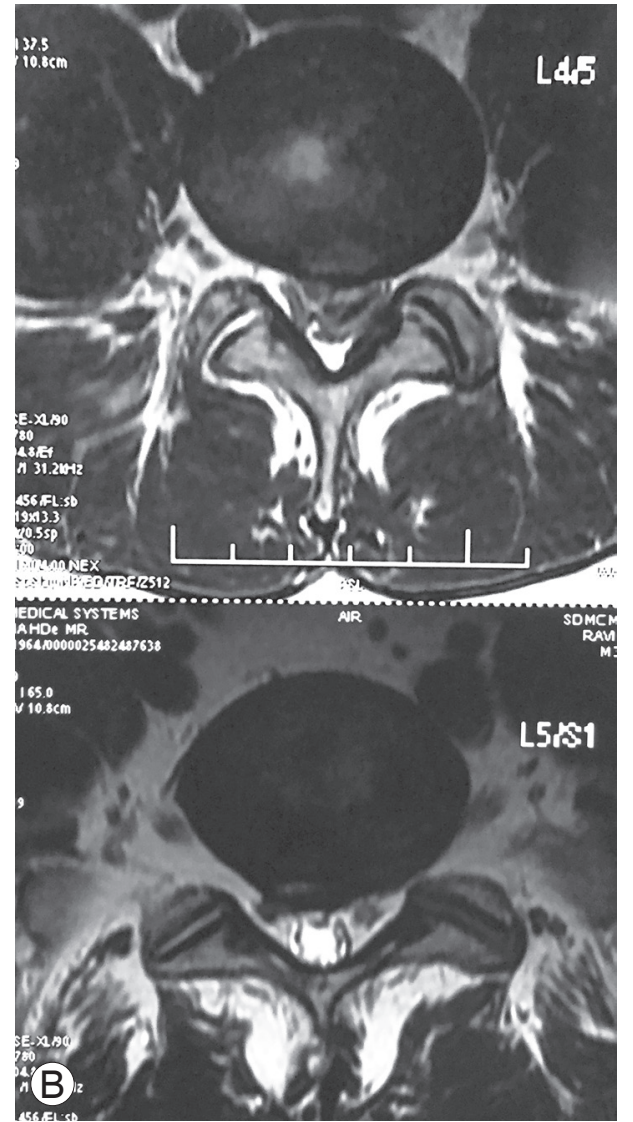


Table 1. Neuro-deficit and its association with various MRI findings

\begin{tabular}{|c|c|c|c|c|}
\hline Serial no. & MRI findings & Subtype & Odds ratio $(95 \% \mathrm{CI})$ & $p$-value \\
\hline \multirow[t]{2}{*}{1} & Position & Central & $0.5625(0.125-2.517)$ & Not significant \\
\hline & & Paracentral & $0.6049(0.225-1.625)$ & Not significant \\
\hline 2 & Degree & & $0.6604(0.192-2.326)$ & Not significant \\
\hline 3 & Migration & & $0.7225(0.289-1.800)$ & Not significant \\
\hline \multirow[t]{2}{*}{4} & Stenosis & Present & 3.3058 (1.167-0.514) & 0.022 \\
\hline & & Absent & $0.1375(0.03-0.514)$ & 0.001 \\
\hline 5 & Type & & 2.78 (0.663-11.623) & Not significant \\
\hline
\end{tabular}

$\mathrm{MRI}$, magnetic resonance imaging; $\mathrm{Cl}$, confidence interval.

correlated with the root involved. The position of the lumbar disc prolapse did not show any significant association with the neurological deficit, with $42.42 \%, 50 \%$, and $75 \%$ of paracentral, postero-central, and foraminal prolapses, respectively, indicating some degree of motor weakness. Three patients walked with a lurch and experienced severe, unbearable pain (visual analogue scale, VAS: 10), and were found to have a foraminal disc prolapse. Various MRI findings such as the position, degree, migration, stenosis, and the duration of symptoms, were analyzed using a logistic regression analysis to determine the odd's ratio and clinical significance for their association with neurological deficits. Table 1 lists the odd's ratio with a 95\% confidence interval and significant $p$-values. A significant association was found between the neural foramen compromise observed via the MRI and the presence of a neurological deficit $(p=0.02)$.

The protrusions $(n=28)$, extrusions $(n=19)$, and sequestrations $(n=4)$ did not have any clinical impact on the presence of a neurological deficit, although the VAS scores for leg pain were higher for sequestrations (mean: 8 for protrusions, 8.8 for extrusions, and 9.2 for sequestrations). The superior and inferior migrations did not have any impact on clinical findings, and the MRI scans provided an accurate intraoperative location and degree of herniation of the fragment in each case.

There were 16 patients (32\%) found to have a highintensity zone (HIZ) on the MRI scan, as indicated by a hyperintense T2 signal at the location of the annular defect. Clinically, these patients had higher VAS scores for
Table 2. Correlation of MRI findings with intraoperative findings

\begin{tabular}{lll} 
Serial no. & MRI findings & $\begin{array}{c}\text { Intraoperative } \\
\text { correlation }\end{array}$ \\
\hline 1 & Position & Complete \\
2 & Levels & $100 \%$ Sensitivity \\
& $94.9 \%$ Specificity \\
\hline 3 & Degree & Complete \\
\hline 4 & Migration & Complete \\
5 & Stenosis & Well appreciated \\
6 & Type & Complete \\
7 & HIZ & $62.5 \%$ identified \\
\hline
\end{tabular}

MRI, magnetic resonance imaging; HIZ, high-intensity zone.

both back $(6.6 \pm 2.12)$ and leg pain (8.8) compared to the other patients (VAS for back, $4.4 \pm 1.04$; leg, 8.6). The VAS for back pain in those with and without HIZ was found to be significant ( $p=0.0001)$. Intraoperatively, a penfield was walked along the annulus to identify the annular defect and it was successfully identifiable in 10 cases. Table 2 provides a summary of the intraoperative correlations for the various parameters studied.

The anatomy of the lateral recess and neural foramen was studied with the goal of predicting the need for a foraminotomy. Neural foramen integrity was classified based on (1) thecal sac compression, (2) nerve root contact, and (3) nerve root compression. Of the 51 levels studied, 24 patients exhibited thecal sac compression in the foramen (17 of which resulted from a hypertrophied 
superior articular facet causing lateral recess stenosis), four patients had nerve root contact, and three had root compression. In the remaining 27 patients without thecal sac compression in the lateral recess, the root was traced intraoperatively into the foramen, it was felt to be normal, and peri-neural fat was observed. All of the patients with a hypertrophied superior facet were found to have a "tight" lateral recess and required undercutting of the facet. The patients with root contact and root compression had reduced/no peri-neural fat and required a foraminotomy to free the entrapped roots.

\section{Discussion}

Few studies have analyzed the correlation between clinical findings and MRI scans for lumbar disc herniation $[6,11,12]$. To our knowledge, the present study is the first to extend this correlation to intraoperative findings. In addition, this is the only study to use a 1.5 Tesla MRI scan, which is currently the preferred resolution among spinal surgeons. As a growing number of spinal surgeons prefer a minimal access discectomy, the dependence on the preoperative assessment (i.e., clinical findings and radiological images), and its correlation with intraoperative findings, is extremely important for successful surgical planning and execution.

The 50 patients included in our study had 56 levels of prolapsed discs. Only surgically significant herniated discs were removed [13] and this was determined to be present at 51 levels by clinical evaluation. There were 44 patients who had a single-level herniation while six patients had a two-level herniation. At our institution, only the herniated fragment is removed when it is symptomatic, and the parent disc is not violated. In accordance with these guidelines, an open discectomy was performed only for symptomatic levels as determined by the clinical examination. Since all the disc prolapses were observed between L3 to S1, it can be inferred that in 50 patients, 56 disc lesions were seen out of a total of 150 discs studied (three per patient: L34, L45, L5S1; not considering L1-2 and L2-3 due to no disc prolapses seen at these levels). Ninety-four levels had no lesions and five were false positives (not clinically significant). Therefore, it can be deduced with respect to determining clinically significant levels that this study revealed the sensitivity of the 1.5 Tesla MRI scan to be $100 \%$ with a specificity of $94.94 \%$. This finding further promotes the fact that all prolapse disc levels observed on an MRI are not all clinically significant levels. Studies [7] have described incorrect level explorations due to the inaccurate level marking of the MRI scan and lumbarization of S1. However, we consider this to be a technical error of marking and interpretation of the images. In this study, no incorrect levels were exposed. This was attributed to the enumeration of the number of mobile discs from the caudal to the cephalad end on the MRI scan preoperatively, and by correlation with an intraoperative X-ray before commencing the procedure.

A positive SLRT is a very important clinical sign for lower lumbar root tension. Multiple studies have corroborated the accuracy of the test and have noted positivity in $86 \%$ to $96 \%$ of the patients [14-18]. While its importance is highly appreciated, its absence does not preclude the presence of a lumbar disc herniation [18]. In this study, a positive SLRT was observed in $74 \%$ of patients, with $75 \%$, $85 \%$, and $43 \%$ for lateral, paracentral, and posterocentral disc prolapse levels, respectively, showing positivity. The logistic regression analysis revealed no significant association between the grade of disc prolapse, migration, or SLRT. A similar finding was reported by Guo et al. [19] wherein they reported that the shape and size of the protruded nucleus pulposus had no parallel relationship with the patient's SLRT.

The position of the disc prolapse correlated well with the intraoperative localization. No correlation was found between the neurological deficits and the degree of herniation, location, migration, levels, and chronicity. However, the logistic regression analysis revealed a significant association between the neural foramen compromise observed on the MRI and the presence of neurological deficits ( $p=0.02$ ). In addition, we found that the absence of lateral recess/foraminal stenosis, irrespective of the other MRI features, had a significant relationship with the absence of neurological deficits $(p=0.001)$. Three patients with a foraminal disc prolapse experienced significant leg pain (VAS 10), neurological deficit, and nerve root compression as shown on the MRI. During surgery, the disc fragment was found to impinge on the dorsal root ganglion in all the three patients. The protrusion, extrusion, and sequestration showed a trend toward an increase in pain, although this did not reach statistical significance. In all cases, the MRI findings were confirmed to be the same as the intraoperative findings. While the morphology of the disc was exactly as expected, the consistency (e.g., hard, soft, boggy, and calcified) was difficult to assess via 
the scans. One sequestered disc was found to be impacted into the dura at the axilla of the nerve root, and it was very challenging to safely separate it for excision. Thus, the degree of difficulty could not have been anticipated by studying the MRI scan.

HIZs have been studied for over 20 years since Aprill and Bogduk [20] first described the concept in 1992, and it is widely believed to be a cause of low back pain. We found similar results in our study as 16 patients exhibited an HIZ on the axial T2 weighted MRI scans. Their mean VAS for low back pain was $6.6 \pm 2.1$ as compared to $4.4 \pm 1.04$ in the other patients, and this finding was significant $(p<0.05)$. An attempt was made to identify these defects during surgery. It was successfully identified in 10 patients, the defect was widened, and the parent disc was explored for fragmentation of the disc material. In the remaining six cases, a small incision in the annulus was made and the disc was explored. In the other 35 discectomies that were performed, the parent disc was not violated.

Janardhana et al. [6] reported difficulty in appreciating the anatomy of the neural foramen using a 0.5-T MRI machine. With the 1.5 Tesla MRI scan, no such difficulty was encountered. The neural foramen was visualized with great clarity and the reporter could easily distinguish between thecal sac compression, root contact, and root compression. Moreover, the MRI observations corroborated well with the intraoperative findings. In addition, in the three cases in which root compression in the foramen was found preoperatively, we noted the absence of perineural fat, considered to be an important intraoperative sign suggestive of chronic nerve compression.

A limitation of this study was the number of patients that fitted the inclusion criteria and were subsequently studied. Similar studies should be conducted using a larger number of patients to observe similar trends and attribute greater significance to the observed findings.

\section{Conclusions}

MRI findings strongly correlate with intraoperative features and can serve as a great tool in planning for a discectomy via minimal access (PELD and tubular-assisted discectomy). While it represents a window into the detailed anatomy of each patient, all observed findings are not necessarily of significance. The decision for surgery should be made only when detailed clinical findings in conjunction with the MRI findings allow for an accurate identification of the culprit fragment and pain generators.

\section{Conflict of Interest}

No potential conflict of interest relevant to this article was reported.

\section{References}

1. Postacchini F. Management of herniation of the lumbar disc. J Bone Joint Surg Br 1999;81:567-76.

2. Vroomen PC, de Krom MC, Wilmink JT. Pathoanatomy of clinical findings in patients with sciatica: a magnetic resonance imaging study. J Neurosurg 2000;92:135-41.

3. Carlisle E, Luna M, Tsou PM, Wang JC. Percent spinal canal compromise on MRI utilized for predicting the need for surgical treatment in single-level lumbar intervertebral disc herniation. Spine J 2005;5:608-14.

4. Greenberg JO, Schnell RG. Magnetic resonance imaging of the lumbar spine in asymptomatic adults. Cooperative study: American Society of Neuroimaging. J Neuroimaging 1991;1:2-7.

5. Jensen MC, Brant-Zawadzki MN, Obuchowski N, Modic MT, Malkasian D, Ross JS. Magnetic resonance imaging of the lumbar spine in people without back pain. N Engl J Med 1994;331:69-73.

6. Janardhana AP, Rajagopal, Rao S, Kamath A. Correlation between clinical features and magnetic resonance imaging findings in lumbar disc prolapse. Indian J Orthop 2010;44:263-9.

7. Lunawat SK, Taneja DK, Malviya A. Correlation between MRI and intra-operative findings in prolapse intervertebral disc. Indian J Orthop 2002;36:12.

8. Boden SD, Wiesel SW. Lumbar spine imaging: role in clinical decision making. J Am Acad Orthop Surg 1996;4:238-48.

9. Boden SD, Davis DO, Dina TS, Patronas NJ, Wiesel SW. Abnormal magnetic-resonance scans of the lumbar spine in asymptomatic subjects: a prospective investigation. J Bone Joint Surg Am 1990;72:403-8.

10. Borenstein DG, O'Mara JW Jr, Boden SD, et al. The value of magnetic resonance imaging of the lumbar spine to predict low-back pain in asymptomatic subjects : a seven-year follow-up study. J Bone Joint Surg Am 2001;83:1306-11. 
11. Rehman L, Khaleeq S, Hussain A, Ghani E, Mushtaq, Khaleeq-uz-Zaman. Correlation between clinical features and magnetic resonance imaging findings in patients with lumbar disc herniation. J Postgrad Med Inst 2007;21:65-70.

12. Beattie PF, Meyers SP, Stratford P, Millard RW, Hollenberg GM. Associations between patient report of symptoms and anatomic impairment visible on lumbar magnetic resonance imaging. Spine (Phila Pa 1976) 2000;25:819-28.

13. Elfering A, Semmer N, Birkhofer D, Zanetti M, Hodler J, Boos N. Risk factors for lumbar disc degeneration: a 5-year prospective MRI study in asymptomatic individuals. Spine (Phila Pa 1976) 2002;27:12534.

14. Jonsson B, Stromqvist B. Significance of a persistent positive straight leg raising test after lumbar disc surgery. J Neurosurg 1999;91:50-3.

15. Lewis PJ, Weir BK, Broad RW, Grace MG. Long-term prospective study of lumbosacral discectomy. J Neurosurg 1987;67:49-53.
16. Jonsson B, Stromqvist B. Neurologic signs in lumbar disc herniation: preoperative affliction and postoperative recovery in 150 cases. Acta Orthop Scand 1996; 67:466-9.

17. Lebkowski WJ. Presence and intensity of the Lasegue sign in relation to the site of lumbar intervertebral disc herniation. Chir Narzadow Ruchu Ortop Pol 2002;67:265-8.

18. Kosteljanetz M, Bang F, Schmidt-Olsen S. The clinical significance of straight-leg raising (Lasegue's sign) in the diagnosis of prolapsed lumbar disc: interobserver variation and correlation with surgical finding. Spine (Phila Pa 1976) 1988;13:393-5.

19. Guo W, Zhao P, Zhou W, et al. Correlation studies between MRI and the symptom scores of patients with LDH before and after manipulative therapy. Zhongguo Gu Shang 2010;23:17-9.

20. Aprill C, Bogduk N. High-intensity zone: a diagnostic sign of painful lumbar disc on magnetic resonance imaging. Br J Radiol 1992;65:361-9. 